\title{
PERIODIC POINTS OF EQUIVARIANT MAPS
}

\author{
JERZY JEZIERSKI and WACŁAW MARZANTOWICZ*
}

\begin{abstract}
We assume that $X$ is a compact connected polyhedron, $G$ is a finite group acting freely on $X$, and $f: X \rightarrow X$ an $G$-equivariant map. We find formulae for the least number of $n$-periodic points in the equivariant homotopy class of $f$, i.e., $\inf _{h} \# \operatorname{Fix}\left(h^{n}\right)$ (where $h$ is $G$-homotopic to $f$ ). As an application we prove that the set of periodic points of an equivariant map is infinite provided the action on the rational homology of $X$ is trivial and the Lefschetz number $L\left(f^{n}\right)$ does not vanish for infinitely many indices $n$ commeasurable with the order of $G$. Moreover, at least linear growth, in $n$, of the number of points of period $n$ is shown.
\end{abstract}

\section{Introduction}

In the study of the dynamics of a self-map $f: X \rightarrow X$ the following invariants of $f$ are considered: the set $P^{n}(f)=\operatorname{Fix}\left(f^{n}\right)$ of points of period $n$ and its cardinality \# $P^{n}(f)$, the set $P_{n}(f)$ of $n$-periodic points, $n$ the minimal period, and its cardinality \# $P_{n}(f)$, the set $P(f)=\bigcup_{n=1}^{\infty} P^{n}(f)$ of all periodic points and its cardinality, the set $\operatorname{Per}(f) \subset \mathrm{N}$ of all minimal periods, finally the rate of growth of \# $P^{n}(f)$.

It was shown in [19] that if $X$ is a compact manifold, $f: X \rightarrow X$ a map and the sequence of Lefschetz numbers of iterations $L\left(f^{n}\right)$ is unbounded then $\# P(f)=\infty$ provided $f$ is a $C^{1}$-map. This is not true for continuous maps in general. Next, in [2] it was proved that in such a case the rate of growth of \# $P^{n}(f)$ is at least linear which improved the previous results. Recently, in [10] a sharper constant of growth for \# $P^{n}(f)$ was derived.

On the other hand since $N\left(f^{n}\right) \leq \# P^{n}(f)$, the rate of growth of the sequence of Nielsen numbers of iterations gives the required estimate for any continuous map. But the Nielsen number $N\left(f^{n}\right)$ is difficult to compute in general (see [15], [16] for detailed expositions). However for continuous maps of some $K(\pi, 1)$-manifolds, $N\left(f^{n}\right)$ can be computed due to the Anosov theorem comparing $N(f)$ with $|L(f)|$. This also gives information about \# $P_{n}(f)$, and permits us to describe $\operatorname{Per}(f)$ by estimating it by its subset consisting of the homotopy minimal periods that are determined by the sequence $N\left(f^{n}\right)$, which thus is a homotopy invariant of $f$ (cf. [13], [18] and [15, Chapt. VI]). For

\footnotetext{
* Both authors supported by Polish National Research Grants PO3A 03929, N N201 373236.

Received 4 August 2009, in final form 22 November 2009.
} 
self-maps of surfaces $N(f)$ can be derived by the use of a generalized Lefschetz number in the sense of Fadell and Husseini which, after an adaption for iterations, also gives information about the rate of growth of $\# P^{n}(f)$ (cf. [17]).

One purpose of this work is to show that the assumption of smoothness $\left(C^{1}\right)$ on $f$ of [2], [19] can be dropped and replaced with the commutativity of $f$ with a free homeomorphism $g: X \rightarrow X$. The paper is a continuation of the previous paper [14] where the case of self-maps $f: S^{d} \rightarrow S^{d}$ of the sphere was studied.

To do it we develop an equivariant Nielsen theory, and an equivariant Nielsen number, for a mapping $f G$-equivariant with respect to a free action of a finite group $G$. Under the assumption that $X$ is a compact connected polyhedron, $G$ is a finite group acting freely on $X$ and $f: X \rightarrow X$ an equivariant map. We define a non-negative integer $N F_{n}^{G}(f)$ (Definition 4.6) and we show that it satisfies the basic properties of the Nielsen number (Theorem 4.8).

1. $N F_{n}^{G}(f)$ is a $G$-homotopy invariant.

2. $N F_{n}^{G}(f) \leq \# \operatorname{Fix}\left(h^{n}\right)$ for any map $h$ in the $G$-homotopy class of $f$.

Our main geometrical result is an equivariant version of the Wecken theorem for periodic points proved by the first author in [11], [12] (see also [15] for a simpler proof). Theorem 4.9 states that if $X$ is a compact manifold of dimension $\geq 3$ then there is a map $h$ which is $G$-homotopic to $f$ and

$$
N F_{n}^{G}(f)=\# \operatorname{Fix}\left(h^{n}\right) .
$$

Next we give some explicit formulae for this invariant under the assumption that $G$ is abelian. Theorem 5.7 says that $N F_{n}^{G}(f)$ grows to infinity if a part of $n$ commeasurable with the order of $G$ (i.e., powers of these primes which appear in the decomposition of \#G) tends to infinity and all the Nielsen classes are essential. These formulae are very transparent when $G$ is an abelian $p$-group (Theorem 5.8).

As an application, in Section 6 we present a general version of the main result of [14]. We show that for any $G$-equivariant map $f: X \rightarrow X$ of a finite polyhedron $X$, or a finite $C W$-complex, with a free action of a finite group $G$ (Proposition 6.1, Corollary 6.2, Theorem 6.3) there are infinitely many minimal periods. Furthermore, as in the $C^{1}$ case, the number of periodic points of period $n$ grows at least linearly in $n$ provided the action of $G$ on homology space $H_{*}(X ; \mathrm{Q})$ is trivial and a condition on sequence $\left\{L\left(f^{n}\right)\right\}_{1}^{\infty}$ is satisfied.

In the last section we consider equivariant maps of products of odd dimensional spheres. We show that an assumption on the spectrum the map implies infinitely many periodic points. 


\section{Nielsen Theory}

We recall briefly some facts about Nielsen theory. For the details we refer the reader to [16].

A few words about the notation. Usually the covering maps are denoted by $p: \tilde{X} \rightarrow X$ and we will do so in this section. However in the rest of the paper we will be given a space $X$ with a free action of a finite group $G$ on $X$. This yields a covering $X \rightarrow \bar{X}=X / G$ onto the orbit space. We will denote this covering $p: X \rightarrow \bar{X}$.

Let $p: \tilde{X} \rightarrow X$ be a universal covering of a polyhedron. We denote by

$$
\mathcal{O}_{X}:=\{\alpha: \tilde{X} \rightarrow \tilde{X}: p \alpha=p\}
$$

the group of deck transformations of this covering. This group has a (noncanonical) bijection with the fundamental group $\pi_{1} X$ although we will not use this correspondence in this paper. Let $f: X \rightarrow X$ be a map and let $\operatorname{lift}(f)=\{\tilde{f}: \tilde{X} \rightarrow \tilde{X}: p \tilde{f}=f p\}$ denote the set of all lifts of $f$. If we fix a lift $\tilde{f}_{0}$, then each other lift of $f$ can be uniquely written as $\alpha \tilde{f}_{0}, \alpha \in \mathscr{O}_{X}$. Consider the action of $\mathcal{O}_{X}$ on the set $\operatorname{lift}(f)$ given by

$$
\alpha \circ \tilde{f}=\alpha \tilde{f} \alpha^{-1} .
$$

The orbits of this action are called Reidemeister classes and their set is denoted by $\mathscr{R}(f)$.

On the other hand we consider the fixed point set:

$$
\operatorname{Fix}(f):=\{x \in X: f(x)=x\} .
$$

We define the Nielsen relation on this set as follows. We say that two fixed points $x, y$ are Nielsen related if there is a path $\omega:[0,1] \rightarrow X$ satisfying: $\omega(0)=x, \omega(1)=y$ and moreover the paths $\omega$ and $f \omega$ are homotopic rel $\{0,1\}$. This relation divides $\operatorname{Fix}(f)$ into a finite number of mutually disjoint classes. A Nielsen class $A$ is called essential if its fixed point index $\operatorname{ind}(f, A) \neq$ 0 . We denote the set of all Nielsen classes by $\mathcal{N}(f)$. It turns out that, for any lift $\tilde{f} \in \operatorname{lift}(f)$, the set $p(\operatorname{Fix}(\tilde{f}))$ is either a Nielsen class of $f$ or is the empty set. Each Nielsen class is of the above form. Moreover there is a natural map $j: \mathscr{N}(f) \rightarrow \mathscr{R}(f)$ which turns out to be injective (but is not onto in general). This map is given by the following correspondence: for a Nielsen class $A \subset \operatorname{Fix}(f)$ we define $j(A)=[\tilde{f}]$ where the lift $\tilde{f} \in \operatorname{lift}(f)$ satisfies $A=p(\operatorname{Fix}(\tilde{f}))$. Thus we may identify each Nielsen class with a Reidemeister class. On the other hand the restriction of $f$ to $\operatorname{Fix}\left(f^{k}\right)$ is a natural homeomorphism which induces the self-map of $\mathcal{N}\left(f^{k}\right)$ which extends to the 
self-map $\mathscr{R}_{f}: \mathscr{R}\left(f^{k}\right) \rightarrow \mathscr{R}\left(f^{k}\right)$ given by $\mathscr{R}_{f}[h]=\left[h^{\prime}\right]$, where $h^{\prime} \in \operatorname{lift}\left(f^{k}\right)$ is the unique lift making the diagram

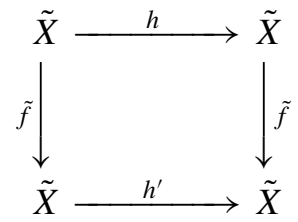

commutative (for a fixed lift $\tilde{f}$ of $f$ ). Since $\left(\mathscr{R}_{f}\right)^{k}=\mathrm{id}$, we get an action of the group $Z_{k}$ on $\mathscr{R}\left(f^{k}\right)$. The orbits of this action are called orbits of Reidemeister classes and their set is denoted by $\mathscr{O} \mathscr{R}\left(f^{k}\right)$. Now we consider the natural map

$$
\operatorname{lift}(f) \ni \tilde{f} \mapsto \tilde{f}^{k} \in \operatorname{lift}\left(f^{k}\right) .
$$

This induces the map $i_{k 1}: \mathscr{R}(f) \rightarrow \mathscr{R}\left(f^{k}\right)$. Similarly we define $i_{k l}$ : $\mathscr{R}\left(f^{l}\right) \rightarrow \mathscr{R}\left(f^{k}\right)$ for $l \mid k$.

Definition 2.1. A Reidemeister class $A \in \mathscr{R}\left(f^{k}\right)$ is called essential if it corresponds to an essential Nielsen class.

A Reidemeister class $A \in \mathscr{R}\left(f^{k}\right)$ is called reducible if $A=i_{k l}(B)$ for $B \in \mathscr{R}\left(f^{l}\right)$, where $l \mid k, l<k$. An orbit of Reidemeister classes is called reducible if one (hence all) of its elements is a reducible Reidemeister class.

The following theorem gives an estimate of the number of k-periodic points.

THEOREM 2.2. For any self-map $f: X \rightarrow X$ of a finite polyhedron and $a$ fixed natural number $k \in \mathrm{N}$

$$
\# \operatorname{Fix}\left(f^{k}\right) \geq \sum_{r \mid k}\left(\# \mathscr{I} \mathscr{E} \mathscr{O} \mathscr{R}\left(f^{r}\right)\right) \cdot r
$$

where $\mathscr{I} \mathscr{O} \mathscr{O}\left(f^{r}\right)$ denotes the set of irreducible $(\mathscr{I})$ essential $(\mathscr{E})$ orbits $(\mathscr{O})$ of Reidemeister $(\mathscr{R})$ classes of the map $f^{r}$.

Proof. The inequality follows from:

(1) each essential Reidemeister class (considered as the Nielsen class) is non-empty,

(2) irreducible Reidemeister classes are mutually disjoint,

(3) each irreducible essential orbit of Reidemeister classes in $\mathscr{I E O C \mathscr { R }}\left(f^{r}\right)$ contains at least $r$ periodic points (of period $r$ ).

In [16] Boju Jiang introduced a number $N F_{k}(f)$, which we will call the Nielsen-Jiang full periodic number. It is a homotopy invariant and is a lower 
bound for the cardinality of $\operatorname{Fix}\left(f^{k}\right)$ :

$$
N F_{k}(f) \leq \# \operatorname{Fix}\left(f^{k}\right)
$$

of the self-map $f: X \rightarrow X$ of a finite polyhedron. We sketch the definition. We consider the disjoint union $\bigcup_{l \mid k} \mathscr{O} \mathscr{R}\left(f^{l}\right)$. For an orbit $A \in \bigcup_{l \mid k} \mathscr{O} \mathscr{R}\left(f^{l}\right)$ we will denote by $l(A)$ the unique divisor of $k$ satisfying $A \in \mathscr{O} \mathscr{R}\left(f^{l(A)}\right)$.

Let $S \subset \bigcup_{l \mid k} \mathscr{O} \mathscr{R}\left(f^{l}\right)$ satisfy: each essential orbit in $\bigcup_{l \mid k} \mathscr{O} \mathscr{R}\left(f^{l}\right)$ can be reduced to an orbit (which may be not essential) in $S$. Then we consider the sums $\sum_{A \in S} l(A)$. The number $N F_{k}(f)$ is defined as the least such sum.

THeORem 2.3 ([15, Thm. 5.3.1, p. 225]). If $M$ is a manifold of dimension at least three then each map $f: M \rightarrow M$ is homotopic to a map $g$ satisfying

$$
\operatorname{Fix}\left(g^{k}\right)=N F_{k}(f)
$$

In general, for a self-map of a compact polyhedron, (see [15, form. (5.1.20), p. 197])

$$
\sum_{r \mid k}\left(\# \mathscr{I} \mathscr{E} \mathscr{O} \mathscr{R}\left(f^{r}\right)\right) \cdot r \leq N F_{k}(f) .
$$

Definition 2.4. We say that a self-map $f: X \rightarrow X$ is essentially reducible if when an essential Nielsen class $A$ reduces to a class $B$ then $B$ is also essential.

Proposition 2.5 ([15, Lemma 5.1.2, p. 198]). If a self-map of a polyhedron $f: X \rightarrow X$ is essentially reducible, then

$$
\sum_{r \mid k}\left(\# \mathscr{I} \mathscr{O} \mathscr{R}\left(f^{r}\right)\right) \cdot r=N F_{k}(f) .
$$

\section{Periodic points of a self-map of the quotient space}

In this section we consider a simply-connected polyhedron $X$ with a free action of a finite group $G$. Let $p: X \rightarrow \bar{X}$ be the natural projection on the quotient, i.e., orbit space $\bar{X}=X / G$. Notice that $p$ is a universal covering. We will give an estimate for the number of periodic points of an equivariant map $f$. Since $p\left(\operatorname{Fix}\left(f^{k}\right)\right) \subset \operatorname{Fix}\left(\bar{f}^{k}\right)$, we first consider the periodic points of the map $\bar{f}$.

We recall some results from [14]. Since it is a statement about a property of a covering map we use the notation of the theory of coverings. 
Lemma 3.1 ([14, Lemma 4.1]). Consider the commutative diagram

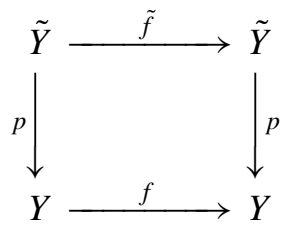

where $p: \tilde{Y} \rightarrow Y$ is a finite regular covering of a finite polyhedron $Y$. Let $\mathcal{O}_{Y}$ be the group of covering transformations of this covering.

Then

$$
\operatorname{ind}(\tilde{f})=r \cdot \operatorname{ind}(f ; p(F i x(\tilde{f})))
$$

where $r=\#\left\{\alpha \in \mathscr{O}_{Y} ; \tilde{f} \alpha=\alpha \tilde{f}\right\}$. In particular $\operatorname{ind}(f ; p(\operatorname{Fix}(\tilde{f}))) \neq 0$ if and only if $L(\tilde{f})=\operatorname{ind}(\tilde{f}) \neq 0$.

Note that in Lemma 3.1 we do not need to assume that the covering $p$ is universal.

REMARK 3.2. Thanks to this lemma, if all Lefschetz numbers $L(\alpha f)$ (where $f$ is an equivariant map and $\alpha$ is a deck transformation) are nonzero, then all Reidemeister classes of $\bar{f}$ are essential. In particular this holds for equivariant self-maps of a sphere if the absolute value of their degree is greater equal 2.

LEMMA 3.3. Let a finite group $G$ act freely on a compact polyhedron $X$. Let $p: X \rightarrow \bar{X}=X / G$ be the natural projection on the quotient space and let $f: X \rightarrow X$ be an equivariant map and $\bar{f}: \bar{X} \rightarrow \bar{X}$ be the induced map. Then

$$
\mathscr{R}(\bar{f})=\text { set of conjugacy classes in } G \text {. }
$$

Proof. Since $f$ is a lift of $\bar{f}$, each other lift is uniquely expressed as $\alpha f$, where $\alpha \in G$. Now

$$
\begin{gathered}
{[\alpha f]=[\beta f] \in \mathscr{R}(\bar{f}) \Longleftrightarrow \exists_{\gamma \in G} \beta f=\gamma \cdot \alpha \cdot f \cdot \gamma^{-1}} \\
\Longleftrightarrow \exists_{\gamma \in G} \beta f=\gamma \cdot \alpha \cdot \gamma^{-1} \cdot f \text { (since } f \text { is equivariant) } \\
\Longleftrightarrow \alpha, \beta \text { represent the same conjugacy class in } G \text {. }
\end{gathered}
$$

LEMmA 3.4. Under the above assumptions, the map $\mathscr{R}_{\bar{f}}: \mathscr{R}\left(\bar{f}^{k}\right) \rightarrow \mathscr{R}\left(\bar{f}^{k}\right)$ is the identity. Therefore each orbit of Reidemeister classes in $\mathscr{R}\left(\bar{f}^{k}\right)$ contains exactly one element. 
Proof. Consider a class $\left[\alpha f^{k}\right] \in \mathscr{R}\left(\bar{f}^{k}\right)$. Then $\mathscr{R}_{\bar{f}}\left[\alpha f^{k}\right]=\left[\beta f^{k}\right]$ where $\beta$ makes the diagram

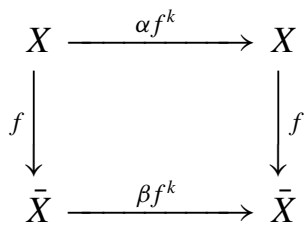

commutative. Since $f$ commutes with $\alpha$, we may put $\beta=\alpha$.

Lemma 3.5. If $G$ is abelian then $\mathscr{O} \mathscr{R}\left(\bar{f}^{k}\right)=\mathscr{R}\left(\bar{f}^{k}\right)=G$.

Corollary 3.6 ([14, Lemma 4.5]). If $\bar{f}: M \rightarrow M\left(M=S^{n} / Z_{m}\right)$ is a map induced by an equivariant map $f: S^{n} \rightarrow S^{n}$, then we have

$$
\# \operatorname{Fix}\left(\bar{f}^{k}\right) \geq \sum_{r \mid k}\left(\# \mathscr{I} \mathscr{R}\left(\bar{f}^{r}\right)\right) \cdot r .
$$

\section{Definition and properties of the lower bound $N F_{n}^{G}(f)$}

We consider a finite polyhedron $X$ with a free action of a finite group $G$ on $X$ and an equivariant map $f: X \rightarrow X$. We look for a lower bound of the cardinality of Fix $\left(g^{n}\right)$ for all $g$ in the $G$-homotopy class of $f$.

Let $p_{X}: X \rightarrow \bar{X}=X / G$ denote the canonical projection on the quotient space. Since the action is free, $p_{X}$ is a covering corresponding to the normal subgroup $p_{X \#}\left(\pi_{1} X\right) \subset \pi_{1} \bar{X}$. This gives rise to the map $\bar{f}$ and the commutative diagram

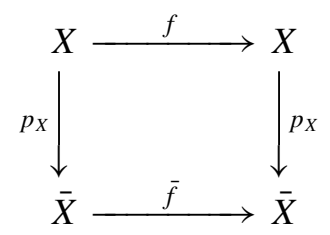

We will identify $G=\left\{\alpha: X \rightarrow X ; p_{X} \alpha=p_{X}\right\}$. To minimize the number of $k$-periodic points of $f$ we will first minimize the corresponding set of $\bar{f}$. Then we will find out which fibres $p_{X}^{-1}(\bar{x})$ (for $\bar{x} \in \operatorname{Fix}\left(\bar{f}^{k}\right)$ ) contain fixed points of $f^{k}$.

Let $\tilde{p}: \tilde{X} \rightarrow X$ be a universal covering. Then the composition $p_{X} \tilde{p}: \tilde{X} \rightarrow$ $\bar{X}$ is also a universal covering. This gives the natural injection $\eta: \operatorname{lift}(f) \rightarrow$ $\operatorname{lift}(\bar{f})$ by the formula $\eta(\tilde{f})=\tilde{f}$, i.e., a lift of $f$ is considered as the lift of $\bar{f}$. The injection $\eta$ induces the map $\eta: \mathscr{R}(f) \rightarrow \mathscr{R}(\bar{f})$.

LEMMA 4.1.

$$
p_{X}(\operatorname{Fix}(f))=\bigcup_{[\tilde{f}] \in \operatorname{im} \eta} p_{X} \tilde{p}(\operatorname{Fix}(\tilde{f}))
$$


is the mutually disjoint union, where in the summation we take one representative from each class $[\tilde{f}] \in \operatorname{im} \eta \subset \mathscr{R}(\bar{f})$.

Proof. Since $\tilde{p}: \tilde{X} \rightarrow X$ is a universal covering, $\operatorname{Fix}(f)=$ $\bigcup_{[\tilde{f}] \in \mathscr{R}(f)} \tilde{p}(\operatorname{Fix}(\tilde{f}))$ is the mutually disjoint union. This implies

$$
p_{X}(\operatorname{Fix}(f))=\bigcup_{[\tilde{f}] \in \mathscr{R}(f)} p_{X}(\tilde{p}(\operatorname{Fix}(\tilde{f}))) .
$$

We notice that

- Each $\tilde{f} \in \operatorname{lift}(f)$ regarded as the lift of $\bar{f}$ represents a class $[\tilde{f}] \in \operatorname{im} \eta \subset$ $\mathscr{R}(\bar{f})$.

- If $\tilde{f}, \tilde{f}^{\prime} \in \operatorname{lift}(f)$ then $p_{X}(\tilde{p}(\operatorname{Fix}(\tilde{f}))) \cap p_{X}\left(\tilde{p}\left(\operatorname{Fix}\left(\tilde{f}^{\prime}\right)\right)\right) \neq \emptyset \Longleftrightarrow[\tilde{f}]=$ $\left[\tilde{f}^{\prime}\right] \in \mathscr{R}(\bar{f})$.

This gives the disjoint union

$$
p_{X}(\operatorname{Fix}(f))=\bigcup_{[\tilde{f}] \in \operatorname{im} \eta} p_{X}(\tilde{p}(\operatorname{Fix}(\tilde{f}))) .
$$

LEMMA 4.2. Under the above notation, let us consider a point $\bar{x}_{0} \in \operatorname{Fix}\left(\bar{f}^{l}\right)$ for some $l \mid k$. Let $\alpha \in G$ satisfy $\bar{x}_{0} \in p_{X}\left(\operatorname{Fix}\left(\alpha f^{l}\right)\right)$. Then the following conditions are equivalent

(1) $p_{X}^{-1}\left(\bar{x}_{0}\right) \cap \operatorname{Fix}\left(f^{k}\right) \neq \emptyset$,

(2) $p_{X}^{-1}\left(\bar{x}_{0}\right) \subset \operatorname{Fix}\left(f^{k}\right)$,

(3) $\alpha^{k / l}=1 \in G$.

Proof. (1) $\Longleftrightarrow(2)$, because $p_{X}^{-1}\left(\bar{x}_{0}\right)$ is an orbit of the action of $G$ and $f^{k}$ is equivariant.

(2) $\Rightarrow$ (3). We assume that $f^{k}(x)=x$ for all $x \in p_{X}^{-1}\left(\bar{x}_{0}\right)$. Moreover $f^{l}(x)=$ $\alpha^{-1}(x)$ for an $x \in p_{X}^{-1}\left(\bar{x}_{0}\right)$. This gives $f^{k}(x)=f^{l}\left(f^{l}\left(\cdots\left(f^{l}(x)\right) \cdots\right)\right)=$ $\alpha^{-k / l}(x)$ and $\alpha^{-k / l}(x)=x$ implies $\alpha^{k / l}=1 \in G$.

(3) $\Rightarrow$ (1) Let $x \in p_{X}^{-1}\left(\bar{x}_{0}\right)$ satisfy $\alpha f^{l}(x)=x$. This implies $f^{l}(x)=\alpha^{-1} x$, hence $f^{k}(x)=f^{l}\left(f^{l}\left(\cdots\left(f^{l}(x)\right) \cdots\right)\right)=\alpha^{-k / l}(x)=x$

Corollary 4.3. Let $\bar{x}_{0} \in p\left(\operatorname{Fix}\left(\alpha f^{l}\right)\right) \subset \operatorname{Fix}\left(\bar{f}^{l}\right)$. Then $\bar{x}_{0} \in p\left(\operatorname{Fix}\left(f^{k}\right)\right)$ $\Longleftrightarrow\left[\bar{x}_{0}\right] \in \operatorname{im} \eta \subset \mathscr{O} \mathscr{R}\left(\bar{f}^{k}\right) \Longleftrightarrow \alpha^{k / l}=1 \in G$.

Proof. By assumption $\bar{x}_{0}=p_{X}\left(x_{0}\right)$ where $x_{0} \in X$ satisfies $\alpha f^{l}\left(x_{0}\right)=x_{0}$. If moreover $\bar{x}_{0} \in p\left(\operatorname{Fix}\left(f^{k}\right)\right)$ then, by Lemma 4.2, $p_{X}^{-1}\left(\bar{x}_{0}\right) \subset \operatorname{Fix}\left(f^{k}\right)$, hence in particular $x_{0} \in \operatorname{Fix}\left(f^{k}\right)$. 
Now $\bar{x}_{0}=p_{X}\left(x_{0}\right) \in p_{X}\left(\operatorname{Fix}\left(f^{k}\right)\right)$ gives $\left[\bar{x}_{0}\right] \in \operatorname{im} \eta \subset \mathscr{O} \mathscr{R}\left(\bar{f}^{k}\right)$. On the other hand the equalities $\alpha f^{l}\left(x_{0}\right)=x_{0}$ and $f^{k}\left(x_{0}\right)=x_{0}$ are equivalent to $\alpha^{k / l}=1$.

Definition 4.4. Let $A \in \mathscr{R}\left(h^{k}\right), B \in \mathscr{R}\left(h^{l}\right)$ be Reidemeister classes. We say that $A$ precedes $B$, and we write $A \preceq B$, if $k \mid l$ and $i_{l k}(A)=B$.

Let $\mathscr{A} \in \mathscr{O} \mathscr{R}\left(h^{k}\right), \mathscr{B} \in \mathscr{O} \mathscr{R}\left(h^{l}\right)$ be Reidemeister classes. We say that $\mathscr{A}$ precedes $\mathscr{B}$, and we write $\mathscr{A} \preceq \mathscr{B}$, if a class in $\mathscr{A}$ precedes a class in $\mathscr{B}$.

Definition 4.5. Let $h: Y \rightarrow Y$ be a self-map of a finite connected polyhedron. Consider the disjoint union $\bigcup_{k \mid n} \mathscr{O} \mathscr{R}\left(h^{k}\right)$. We fix subsets $\mathscr{A}, \mathscr{B} \subset$ $\bigcup_{k \mid n} \mathscr{O} \mathscr{R}\left(h^{k}\right)$. We say that $\mathscr{A}$ precedes $\mathscr{B}$ if for each essential $B \in \mathscr{B}$ there is an element $A \in \mathscr{A}$ preceding $B(A \preceq B)$. We will also say that $\mathscr{A}$ is a preceding system.

For a subset $\mathscr{A} \subset \bigcup_{k \mid n} \mathscr{O} \mathscr{R}\left(h^{k}\right)$ we will consider the sum

$$
\sum_{A \in \mathscr{A}} l(A)
$$

where $A \in \mathscr{O} \mathscr{R}\left(h^{l(A)}\right)$.

We will apply Definition 4.5 to the map $\bar{f}: \bar{X} \rightarrow \bar{X}$ where $\mathscr{B}$ will be equal to

$$
i_{n *}^{-1}[\operatorname{im} \eta] \stackrel{\text { def }}{=} \bigcup_{k \mid n}\left\{A \in \mathscr{O} \mathscr{R}\left(\bar{f}^{k}\right): i_{n k}(A) \in \operatorname{im} \eta \subset \mathscr{O} \mathscr{R}\left(\bar{f}^{n}\right)\right\}
$$

Now we are in a position to define the Nielsen-type number of an equivariant map $f$ (with respect to a free action) as

DEFINITION 4.6.

$$
N F_{n}^{G}(f)=\left(\min _{\mathscr{A}} \sum_{A \in \mathscr{A}} l(A)\right) \cdot \# G
$$

where $\mathscr{A}$ runs over the family of all subsets of $\bigcup_{k \mid n} \mathscr{O} \mathscr{R}\left(\bar{f}^{k}\right)$ preceding $i_{n *}^{-1}[\operatorname{im} \eta]$.

Remark 4.7. A preceding system realizing the minimum, in the above definition, will be called minimal preceding system.

The main geometric property of $N F_{n}^{G}(f)$ is the following.

THEOREM 4.8.

(1) $N F_{n}^{G}(f)$ is a G-homotopy invariant.

(2) $N F_{n}^{G}(f) \leq \# \operatorname{Fix}\left(g^{n}\right)$ for any map $g$ in the G-homotopy class of $f$. 
Proof. (1) is obvious, since the sets of Reidemeister classes and the preceding relation are $G$-homotopy invariants.

Now we prove (2). By virtue of (1) it is enough to prove (2) for $g=f$. If $\operatorname{Fix}\left(f^{n}\right)$ is infinite then the inequality is obvious. We assume that $\operatorname{Fix}\left(f^{n}\right)$ is finite. We consider the set of all orbits of points in $p\left(\operatorname{Fix}\left(f^{n}\right)\right)$. Let $\sigma$ be an orbit of length $l_{\sigma}$. It determines an element $A_{\sigma} \in \mathscr{O} \mathscr{R}\left(\bar{f}^{l_{\sigma}}\right)$.

We will show that the set of orbits of Reidemeister classes

$$
\mathscr{A}=\left\{A_{\sigma} ; \sigma \text { is an orbit in } p\left(\operatorname{Fix}\left(f^{n}\right)\right)\right\} \subset \bigcup_{l \mid n} \mathscr{O} \mathscr{R}\left(\bar{f}^{l}\right)
$$

precedes $i_{n *}^{-1}[\mathrm{im} \eta]$

Let $A \in i_{n *}^{-1}[\mathrm{im} \eta]$ be essential, i.e., $A \in \mathscr{O} \mathscr{R}\left(\bar{f}^{k}\right)$, for some $k \mid n$, is essential and $i_{n k}(A) \in \operatorname{im} \eta$. This implies $A \subset p\left(\operatorname{Fix}\left(f^{n}\right)\right)$ (Corollary 4.3). Since $A$ is essential, it contains an orbit of points $a$ of length $l \mid k$. Now $a \subset p\left(\operatorname{Fix}\left(f^{n}\right)\right)$, hence it defines $A_{a} \in \mathscr{A}, A_{a} \in \mathscr{O} \mathscr{R}\left(f^{l}\right)$. It is evident that $A_{a}$ precedes $A$, since $l \mid k$ and both $A_{a}$ and $A$ contain the orbit $a$. This proves that $\mathscr{A}$ precedes $i_{n *}^{-1}[\operatorname{im} \eta]$.

On the other hand we notice that $p_{X}^{-1}(\sigma)$ contains exactly $l_{\sigma} \cdot \# G$ points and all these points belong to $p\left(\operatorname{Fix}\left(f^{n}\right)\right)($ Lemma 4.2).

Now a sum in the definition of $N F_{n}^{G}(f)$ equals

$$
\left(\sum_{\sigma} l\left(A_{\sigma}\right)\right) \cdot \# G=\left(\sum_{\sigma} l_{\sigma}\right) \cdot \# G=\sum_{\sigma} \# p_{X}^{-1}(\sigma)=\# \operatorname{Fix}\left(f^{n}\right)
$$

where $\sigma$ runs through the family of orbits of points in $p_{X}\left(\operatorname{Fix}\left(f^{n}\right)\right)$. Now $N F_{n}^{G}(f)$ as minimum of (such sums) $\times \# G$ must be $\leq \# \operatorname{Fix}\left(f^{n}\right)$.

THEOREM 4.9 (Wecken Theorem for periodic points of $G$-equivariant maps). Let $X$ be a manifold of dimension at least 3 with a free action of a finite group $G$. Then every G-equivariant map $f$ is $G$-equivariantly homotopic to a map $g$ so that

$$
\# \operatorname{Fix}\left(g^{k}\right)=N F_{k}^{G}(f) .
$$

Proof. We already know (Corollary 4.3) that each $G$-equivariant map $f$ : $X \rightarrow X$ induces $\bar{f}: \bar{X} \rightarrow \bar{X}$ and

$$
\# \operatorname{Fix}\left(f^{n}\right)=\left(\#\left\{\bar{x} \in \operatorname{Fix}\left(\bar{f}^{n}\right) ; i_{n, l(\bar{x})}[\bar{x}] \in \operatorname{im} \eta\right\}\right) \cdot \# G
$$

Splitting the set Fix $\left(\bar{f}^{n}\right)$ into orbits we get

$$
\# \operatorname{Fix}\left(f^{n}\right)=\left(\sum_{a} l(a)\right) \cdot \# G
$$


where $a$ runs through the set of all orbits in $\operatorname{Fix}\left(\bar{f}^{n}\right)$ satisfying $i_{n, l([\bar{a}])}[a] \subset$ $\operatorname{im} \eta$.

On the other hand by definition

$$
N F_{n}^{G}(f)=\left(\min _{\mathscr{A}} \sum_{A \in \mathscr{A}} l(A)\right) \cdot \# G
$$

where $\mathscr{A}$ runs through the family of all subsets in $\bigcup_{l \mid k} \mathscr{O R}\left(f^{l}\right)$ preceding $i_{n *}^{-1}[\operatorname{im} \eta] \subset \bigcup_{l \mid k} \mathscr{O R}\left(f^{l}\right)$.

The theorem will be proved if for a preceding set $\mathscr{A}$ (from the definition of $\left.N F_{n}^{G}(f)\right)$ realizing the least sum, we can deform $\bar{f}$ to a map $\bar{g}$ satisfying:

each orbit of points a of length $l$ in $\operatorname{Fix}\left(\bar{g}^{n}\right)$ satisfying $i_{n, l}([a]) \in \operatorname{im} \eta$ represents a class $A_{a} \in \mathscr{O} \mathscr{R}\left(\bar{g}^{l}\right) \cap \mathscr{A}$ and no other periodic point represents $A_{a}$.

This gives an injection from the set of orbits of points in $\operatorname{Fix}\left(\bar{g}^{n}\right)$ to $\mathscr{A}$ which sends an orbit (of points) of length $l$ to an irreducible class $A_{a} \in \mathscr{O} \mathscr{R}\left(\bar{g}^{l}\right)$.

Now we compare (3) (for $f=g$ ) with 4 and we get the inequality $\sum_{a} l(a) \leq$ $\sum_{A \in \mathscr{A}} l(A)$. Since the reverse inequality is obvious (Theorem 4.8 ), we get

$$
\# \operatorname{Fix}\left(g^{n}\right)=\left(\sum_{a} l(a)\right) \cdot \# G=\left(\sum_{A \in \mathscr{A}} l(A)\right) \cdot \# G=N F_{n}^{G}(f) .
$$

The required deformation $\bar{g}$ is obtained from the Wecken theorem for Periodic Points [12]. In fact a map, of a manifold of dimension at least 3 , realizing the least number of periodic points has the above property for all periodic points (see Lemma 5.3 and the first sentence of the proof of Theorem 3.1 in [11]).

Now we consider the case when $\bar{f}$ is essentially reducible. Then one can check that (see [15])

(1) The family $\mathscr{A}=\left(\bigcup_{l \mid n} \mathscr{I} \mathscr{C} \mathscr{O} \mathscr{R}\left(\bar{f}^{l}\right)\right) \cap i_{n *}^{-1}[\mathrm{im} \eta]$ precedes the set of all essential orbits in $i_{n *}^{-1}[\operatorname{im} \eta]$.

(2) $\mathscr{A}$ is contained in any family of subsets of $\bigcup_{l \mid n} \mathscr{O} \mathscr{R}\left(\bar{f}^{l}\right)$ preceding the set of all essential orbits in $i_{n *}^{-1}[\operatorname{im} \eta]$.

Thus we get

Corollary 4.10. Let $X$ be a finite polyhedron with a free action of a finite group $G$ and $f: X \rightarrow X$ be a G-equivariant self-map of $X$ such that the 
induced map $\bar{f}$ is essentially reducible (for $l \mid k$ ). Then

$$
N F_{k}^{G}(f)=\left(\sum_{l \mid k}\left(\mathscr{\mathscr { C } \mathscr { O } O \mathscr { R }}\left(\bar{f}^{l}\right) \cap i_{n *}^{-1}[\mathrm{im} \eta]\right) \cdot l\right) \cdot(\# G) .
$$

REMARK 4.11. We would like to emphasize that our equivariant full NielsenJiang periodic number is constructed in a different way than an equivariant Nielsen fixed point number introduced in [5] by E. Fadell and P. Wong and developed later by the second author (see [21], and [6] for more references). Also our invariant is based on a different geometric approach than that introduced and used by D. Ferrario [7], [8]. Futhermore it is based on a different approach than that studied by J. Weber [20] for an action of a discrete group. Our Nielsen-Jiang periodic number is defined only for a free action of finite group, but on the other hand it is relatively easy to derive and provides the periodic Wecken theorem for an equivariant map. Moreover to estimate this Nielsen-Jiang periodic number, i.e., to verify the essentiality of the Reidemeister classes of iterations of $f$, we use the classical Lefschetz numbers of these iterations which are easier to calculate.

\section{Explicit Formulae}

Now we will derive formulae for $N F_{k}^{G}(f)$. We consider a simply-connected polyhedron $X$, a free action of a finite abelian group $G$ on $X$ and an equivariant self-map $f: X \rightarrow X$. Again let $\bar{f}: \bar{X} \rightarrow \bar{X}$ denote the induced map of the quotient space. We assume that all the Reidemeister classes are essential.

Since $\pi_{1} \bar{X}$ is finite and abelian it admits a decomposition

$$
G=\pi_{1} \bar{X}=\left(Z_{p_{1}^{a_{11}}} \oplus \cdots \oplus \mathbf{Z}_{p_{1}^{a_{1 u_{1}}}}\right) \oplus \cdots \oplus\left(\mathrm{Z}_{p_{r}^{a_{r 1}}} \oplus \cdots \oplus \mathbf{Z}_{p_{r}^{a_{r} u_{r}}}\right)
$$

where $p_{1}, \ldots, p_{r}$ are distinct primes. We recall that in our case the Reidemeister action is trivial, $\mathscr{R}\left(\bar{f}^{l}\right)=\pi_{1} X$ for all $l$, and each orbit of Reidemeister classes consists of one element, hence $\mathscr{O} \mathscr{R}\left(\bar{f}^{k}\right)=\mathscr{R}\left(\bar{f}^{k}\right)=G$. In particular this implies $\mathscr{\mathscr { E }} \mathscr{O} \mathscr{R}\left(f^{k}\right)=\mathscr{I} \mathscr{R}\left(f^{k}\right)$.

REMARK 5.1. Under the above assumptions

$$
\begin{aligned}
N F_{n}^{G}(f) & =\left(\sum_{k \mid n} \#\left(\mathscr{\mathscr { R }}\left(f^{k}\right) \cap i_{n k}^{-1}(0)\right) \cdot k\right) \cdot m \\
& =\left(\sum_{k \mid n} \#\left(\text { irreducible elements in } i_{n k}^{-1}(0)\right) \cdot k\right) \cdot m .
\end{aligned}
$$


We fix isomorphisms $G=\mathscr{R}\left(\bar{f}^{k}\right)=\mathscr{O} \mathscr{R}\left(\bar{f}^{k}\right)$ by the correspondence

$$
G \ni[\alpha] \rightarrow\left[\alpha \cdot f^{k}\right] \in \operatorname{lift}\left(\bar{f}^{k}\right) .
$$

Now $i_{k l}(x)=k / l \cdot x$, since $i_{k l}[\alpha f]=\left[(\alpha f)^{k / l}\right]=\left[\alpha^{k / l} \cdot f^{k / l}\right]$.

We need a notion we have already used in [14].

DeFINITION 5.2. We say that a natural number $k$ eventually divides $m$ if there exists $c \in \mathrm{N}$ such that $k \mid m^{c}$. In other words $k$ eventually divides $m=p_{1}^{a_{1}} \ldots p_{r}^{a_{r}}, a_{i}>0$ if $k=p_{1}^{b_{1}} \ldots p_{r}^{b_{r}}$, for some $b_{i} \geq 0$.

We start with a general remark that in our case it is enough to find formulae for $N F_{n}^{G}(f)$ for $n$ eventually dividing $m$. For given $m, k \in \mathrm{N}$ let $k^{\prime}$ be the greatest divisor of $k$ which eventually divides $m$ (it can be equal to 1 !).

LEMma 5.3. Under the assumptions of this section $N F_{k}^{G}(f)=N F_{k^{\prime}}^{G}(f)$ where $k^{\prime}$ denotes the greatest divisor of $k$ eventually dividing $m$.

PRoOF. We show that if $k$ does not eventually divide $m$ then all elements in $\mathscr{O} \mathscr{R}\left(f^{k}\right)=\mathscr{R}\left(f^{k}\right)=G$ are reducible. In fact then there exists a prime number $p \mid k$ which does not divide $m$. Now the map $i_{k, k / p}: \bigoplus Z_{p_{s}} a_{i j} \rightarrow \bigoplus Z_{p_{s}}^{a_{i j}}=G$, which is given by $i_{k, k / p}\left[x_{i j}\right]=\left[p \cdot x_{i j}\right]$, is an isomorphism of each summand, since $p$ is relatively prime to any $p_{s}$. Now

$$
\begin{aligned}
N F_{n}^{G}(f) & =\left(\sum_{k \mid n} \#\left(\text { irreducible elements in } i_{n k}^{-1}(0)\right) \cdot k\right) \cdot m \\
& =\left(\sum_{k \mid n^{\prime}} \#\left(\text { irreducible elements in } i_{n k}^{-1}(0)\right) \cdot k\right) \cdot m \\
& =\left(\sum_{k \mid n^{\prime}} \#\left(\text { irreducible elements in } i_{n^{\prime} k}^{-1}(0)\right) \cdot k\right) \cdot m \\
& =N F_{n^{\prime}}^{G}(f)
\end{aligned}
$$

since $i_{n k}=i_{n n^{\prime}} i_{n^{\prime} k}$ and $i_{n n^{\prime}}$ is a bijection.

From now on we will assume that $k$ (hence also any divisor $l$ of $k$ ) eventually divides $m$. We denote $k=p_{1}^{\sigma_{1}} \cdots p_{r}^{\sigma_{r}}, l=p_{1}^{\tau_{1}} \cdots p_{r}^{\tau_{r}}$ where $\sigma_{i} \geq \tau_{i} \geq 0$. We also denote $x_{i j}=p_{i}^{c_{i j}} \cdot x_{i j}^{\prime}$ where $x_{i j}^{\prime}$ is an integer not divisible by $p_{i}$ : for $x_{i j}=0$ we put $c_{i j}=\infty$.

Lemma 5.4. An element $\left[x_{i j}\right] \in \mathscr{R}\left(\bar{f}^{l}\right)=G$, (where $x_{i j} \in \mathrm{Z}_{p_{i}}^{a_{i j}}$ )

(1) represents an irreducible class $\Longleftrightarrow(l=1)$ or (for each $i$ there exists $a j=1, \ldots, u_{i}$ such that $p_{i}$ does not divide $\left.x_{i j}\right)$. 
(2) satisfies $i_{k l}\left[x_{i j}\right]=[0] \Longleftrightarrow p_{i}^{a_{i j}}$ divides $k / l \cdot x_{i j}$ for all $i, j$.

Corollary 5.5. $\left[x_{i j}\right] \in \mathscr{R}\left(\bar{f}^{l}\right)$ is a class which is irreducible and $i_{k l}\left[x_{i j}\right]=$ $[0] \Longleftrightarrow$

(1) $(l=1)$ or (for each $i$ there exists a $j$ such that $\left.c_{i j}=0\right)$,

(2) $c_{i j} \geq a_{i j}-\sigma_{i}+\tau_{i}$ for all $i, j$.

In the next lemma we give the formulae of $N F_{k}^{G}(f)$ in some special cases. Since in the formula of $N F_{k}^{G}(f)$ the factor $m$ is constant, in the computations below we will concentrate on the remaining factor.

Lemma 5.6. Let $G=\mathrm{Z}_{m}$ act freely on a simply-connected polyhedron, where $m=p^{a}$, $p$ is a prime, and let $k=p^{\sigma}$ for some integer $\sigma$. Then

$$
N F_{k}^{G}(f)=k \cdot m \text {. }
$$

Proof. Let us fix $l \mid k$. We have to determine how many classes $[x] \in \mathscr{R}\left(f^{l}\right)$ satisfy the two conditions of Corollary 5.5.

First we consider the case $\sigma \geq a$, i.e., $m \mid k$.

If $l=1$ then each element in $x \in \mathscr{R}\left(f^{l}\right)$ is irreducible and moreover $m \mid k$ implies that $i_{k 1}[x]=[k x]=[0] \in Z_{m}$.

Let $1 \neq l \mid k$. Now $[x] \in \mathscr{R}\left(f^{l}\right)$ is reducible iff $p \mid x$, hence there are exactly $p^{\sigma-1}$ reducible, hence $p^{a}-p^{a-1}$ irreducible elements in $\mathscr{R}\left(f^{l}\right)$. On the other hand we notice that for each irreducible element $[x] \in \mathscr{R}\left(f^{l}\right), p$ does not divide $x=p^{c} x^{\prime}$, hence $c=0$. Now the class $[x] \in \mathscr{R}\left(f^{l}\right)$ satisfies $i_{k l}[x]=$ $[0] \Longleftrightarrow 0 \geq a-\sigma+\tau \Longleftrightarrow \tau \leq \sigma-a$.

Now the summation in the formula in Remark 5.1 is reduced to $l=p^{\tau}$ for $\tau \leq \sigma-a$ and the sum becomes

$$
\begin{aligned}
p^{a}+\sum_{\tau=1}^{\sigma-a}\left(p^{a}-p^{a-1}\right) p^{\tau} & =p^{a}+\left(p^{a}-p^{a-1}\right) \sum_{\tau=1}^{\sigma-a} p^{\tau} \\
& =p^{a}+\left(p^{a}-p^{a-1}\right) \frac{p^{\sigma-a+1}-p}{p-1} \\
& =p^{a}+p^{a}\left(p^{\sigma-a}-1\right)=p^{\sigma}=k .
\end{aligned}
$$

Now we consider the case $\sigma \leq a$, i.e., $k \mid m$.

Let $l=1$. Then all classes are irreducible. Moreover again denoting $x=$ $p^{c} x^{\prime}$ (where $p$ does not divide $x^{\prime}$ ) we have

$$
i_{k 1}[x]=[k x]=\left[p^{\sigma} \cdot p^{c} \cdot x^{\prime}\right]=\left[p^{\sigma+c} \cdot x^{\prime}\right] \in \mathscr{R}\left(f^{k}\right)=\mathrm{Z}_{p^{a}} .
$$


Now $i_{k 1}[x]=[0] \in \mathrm{Z}_{p^{a}} \Longleftrightarrow p^{a} \mid p^{\sigma+c} \Longleftrightarrow a \leq \sigma+c \Longleftrightarrow c \geq a-\sigma$. The last means that $x=p^{a-\sigma} \cdot x^{\prime \prime}$ and there are exactly $p^{\sigma}$ such elements in $Z_{p^{a}}$.

Now we consider $1 \neq l \mid k$ and we denote $l=p^{\tau}$. A class $[x] \in \mathscr{R}\left(f^{l}\right)$ is irreducible $\Longleftrightarrow p$ does not divide $x$. Thus for an irreducible $[x]$

$$
i_{k l}[x]=\left[p^{\sigma} / p^{\tau} \cdot x\right]=\left[p^{\sigma-\tau} \cdot x\right] \neq[0] \in \mathrm{Z}_{p^{a}},
$$

since $\sigma-\tau<\sigma \leq a$. Thus for $l \neq 1$ there is no contribution to the sum, so we get

$$
\sum_{l \mid k} \sum_{x} l=\sum_{l=1} \sum_{x} l=p^{\sigma} \cdot 1=k .
$$

In both cases $N F_{n}(f)=\left(\sum\right) \cdot m=k m$.

Theorem 5.7. Let $G=\pi_{1} X=\mathrm{Z}_{p_{1}^{a_{1}}} \oplus \cdots \oplus \mathrm{Z}_{p_{r}^{a_{r}}}$, where $p_{1}, \ldots, p_{r}$ denote distinct primes, be a cyclic group of order $m=p_{1}^{a_{1}} \ldots p_{r}^{a_{r}}$. Then for $k$ eventually dividing $m$

$$
N F_{k}^{G}(f)= \begin{cases}k m & \text { if } m \mid k, \\ \operatorname{gcd}(m, k) \cdot m & \text { otherwise. }\end{cases}
$$

PRoOF. We again denote $m=p_{1}^{a_{1}} \ldots p_{r}^{a_{r}}, k=p_{1}^{\sigma_{1}} \ldots p_{r}^{\sigma_{r}}, l=p_{1}^{\tau_{1}} \ldots p_{r}^{\tau_{r}}$

Then, by Corollary 5.5, an element $x=\left[x_{1}, \ldots, x_{r}\right] \in \mathbf{Z}_{p_{1}^{a_{1}}} \oplus \cdots \oplus \mathbf{Z}_{p_{r}^{a_{r}}}=$ $\mathscr{R}\left(f^{l}\right)$

(1) is irreducible $\Longleftrightarrow(l=1)$ or (for each $i=1, \ldots, r, \tau_{i} \neq 0$ implies $p_{i}$ does not divide $x_{i}$ ),

(2) $i_{k l}[x]=[0] \in \mathscr{R}\left(f^{k}\right) \Longleftrightarrow \tau_{i}-c_{i} \leq \sigma_{i}-a_{i}$.

We consider two cases. First we assume that $m \mid k$, i.e., $\sigma_{i} \geq a_{i}$ for all $i=$ $1, \ldots, r$. How many irreducible elements belong to $\operatorname{ker}\left(i_{k l}\right)$ ?

If $l=1$ then all the elements in $\mathscr{R}\left(f^{1}\right)=G$ are irreducible and because

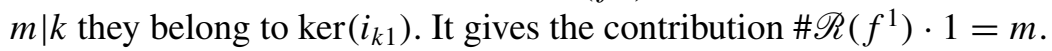

Now $l \neq 1$ and we consider an element $\left[x_{1}, \ldots, x_{r}\right] \in \mathscr{I} \mathscr{R}\left(f^{l}\right) \cap \operatorname{ker}\left(i_{k l}\right)$. Suppose that $\tau_{i} \neq 0$. Now (1) implies $c_{i}=0$ and then (2) becomes $\tau_{i} \leq \sigma_{i}-a_{i}$ for all $i=1, \ldots, r$. Now suppose that $\tau_{i}=0$. Then (1) and (2) hold (since $\sigma_{i} \geq a_{i}$ by the assumption), hence there are no restriction on $x_{i}$. Summing up we get:

$$
\#\left(\mathscr{I} \mathscr{R}\left(f^{l}\right) \cap \operatorname{ker}\left(i_{k l}\right)\right)=m\left(1-\frac{1}{p_{i_{1}}}\right) \ldots\left(1-\frac{1}{p_{i_{s}}}\right)
$$

where $\left(i \in\left\{i_{1}, \ldots, i_{s}\right\}\right.$ iff $\left.\tau_{i} \neq 0\right)$ and $\left(\tau_{i} \leq \sigma_{i}-a_{i}\right.$ for $i \in\left\{i_{1}, \ldots, i_{s}\right\}$. Otherwise \#( $\left.\left.\mathscr{\mathscr { R }}\left(f^{l}\right) \cap \operatorname{ker}\left(i_{k l}\right)\right)=0\right)$. 
Let us notice that (1), implies $\mathscr{I} \mathscr{R}\left(f^{1}\right)=G$ and for $l=p^{\tau}>1$,

$$
\# \mathscr{S} \mathscr{R}\left(f^{l}\right)=m\left(1-\frac{1}{p_{i_{1}}}\right) \ldots\left(1-\frac{1}{p_{i_{s}}}\right)
$$

where $\tau_{i} \neq 0$ iff $i \in\left\{i_{1}, \ldots, i_{s}\right\}$.

Now we split the sum as

$$
\sum_{l \mid k} \sum_{x} l=\sum_{0}+\sum_{1}+\cdots+\sum_{r}
$$

where in $\sum_{s}$ the summation runs through the set $\left\{l=p_{i_{1}}^{\tau_{i_{1}}} \ldots p_{i_{s}}^{\tau_{i_{s}}} ; \tau_{i_{j}} \neq 0\right\}$ (numbers divisible by exactly $s$ prime divisors of $k$ ).

Then $\sum_{0}=m$ while for $s \geq 1$

$$
\begin{aligned}
\sum_{s} & =\sum_{i_{1}, \ldots, i_{s}}\left(\sum_{\tau_{i_{1}}=1}^{\sigma_{i_{1}}-a_{i_{1}}} \ldots \sum_{\tau_{i_{s}}=1}^{\sigma_{i_{s}}-a_{i_{s}}} m \cdot\left(1-\frac{1}{p_{i_{1}}}\right) \ldots\left(1-\frac{1}{p_{i_{s}}}\right) p_{i_{1}}^{\tau_{i_{1}}} \ldots p_{i_{s}}^{\tau_{i_{s}}}\right) \\
& =m \sum_{i_{1}, \ldots, i_{s}}\left(\sum_{\tau_{i_{1}}=1}^{\sigma_{i_{1}}-a_{i_{1}}}\left(1-\frac{1}{p_{i_{1}}}\right) p_{i_{1}}^{\tau_{i_{1}}} \ldots \sum_{\tau_{i_{s}}=1}^{\sigma_{i_{s}}-a_{i_{s}}}\left(1-\frac{1}{p_{i_{s}}}\right) p_{i_{s_{s}}}^{\tau_{i_{s}}}\right) \\
& =m \sum_{i_{1}, \ldots, i_{s}}\left(1-\frac{1}{p_{i_{1}}}\right) \frac{p_{i_{1}}^{\sigma_{i_{1}}-a_{i_{1}}+1}-p_{i_{1}}}{p_{i_{1}}-1} \ldots\left(1-\frac{1}{p_{i_{s}}}\right) \frac{p_{i_{s}}^{\sigma_{i_{s}}-a_{i_{s}}+1}-p_{i_{s}}}{p_{i_{s}}-1} \\
& =m \sum_{i_{1}, \ldots, i_{s}}\left(p_{i_{1}}^{\sigma_{i_{1}}-a_{i_{1}}}-1\right) \ldots\left(p_{i_{s}}^{\sigma_{i_{s}}-a_{i_{s}}}-1\right)
\end{aligned}
$$

and thus

$$
\begin{aligned}
\sum & =\sum_{0}+\sum_{1}+\cdots+\sum_{r}=m \cdot \sum_{I \subset\{1, \ldots, r\}}\left(\prod_{i \in I}\left(p_{i}^{\sigma_{i}-a_{i}}-1\right)\right) \\
& =m\left(1+\left(p_{1}^{\sigma_{1}-a_{1}}-1\right)\right) \ldots\left(1+\left(p_{r}^{\sigma_{r}-a_{r}}-1\right)\right) \\
& =m p_{1}^{\sigma_{1}-a_{1}} \ldots p_{r}^{\sigma_{r}-a_{r}}=p_{1}^{\sigma_{1}} \ldots p_{r}^{\sigma_{r}}=k .
\end{aligned}
$$

Now we consider the case when $m$ does not divide $k$, i.e., $a_{i} \geq \sigma_{i}$ for an $i=1, \ldots, r$. We will show that $\#\left(\mathscr{R}\left(f^{1}\right) \cap i_{n 1}^{-1}(0)\right)=\operatorname{gcd}(k, m)$ and all the classes in $i_{n l}^{-1}(0)$ are reducible for $l \neq 1$. This will give the formula.

We may assume that $a_{i}>\sigma_{i}$ for $i=1, \ldots, s$ (for an $1 \leq s \leq r$ ) and $a_{i} \leq \sigma_{i}$ for $i=s+1, \ldots, r$. 
Let $l=1$. Then all the classes in $\mathscr{R}\left(f^{1}\right)$ are irreducible. We write $[x]=$ $\left[p_{1}^{c_{1}} x_{1}^{\prime}, \ldots, p_{r}^{c_{r}} x_{r}^{\prime}\right]$ where $p_{i}$ does not divide $x_{i}^{\prime}$. Then

$$
i_{k 1}[x]=\left[p_{1}^{\sigma_{1}+c_{1}} x_{1}^{\prime \prime}, \ldots, p_{r}^{\sigma_{r}+c_{r}} x_{r}^{\prime \prime}\right]
$$

where $p_{i}$ does not divide $x_{i}^{\prime \prime}$. Now $i_{k 1}[x]=[0] \in \mathscr{R}\left(f^{k}\right) \Longleftrightarrow p_{i}^{a_{i}} \mid p_{i}^{\sigma_{i}+c_{i}} \Longleftrightarrow$ $c_{i} \geq a_{i}-\sigma_{i}$. Now for $i=1, \ldots, s$ we get $p_{i}^{a_{i}-\sigma_{i}} \mid x_{i}$ and we have exactly $p_{i}^{a_{i}} / p_{i}^{a_{i}-\sigma_{i}}=p_{i}^{\sigma_{i}}$ such elements in $\mathscr{R}\left(f^{k}\right)$.

On the other hand for $i=s+1, \ldots, r$ we have $a_{i}-\sigma_{i} \leq 0$ hence the inequality is satisfied for each $x_{i}$. This implies that $\# i_{k 1}^{-1}[0]=p_{1}^{\sigma_{1}} \ldots p_{s}^{\sigma_{s}} p_{s+1}^{a_{s+1}} \ldots p_{r}^{a_{r}}=$ $\Pi_{i=1}^{r} p_{i}^{\min \left(\sigma_{1}, a_{i}\right)}$.

Now we consider the case $l \neq 1, l \mid k$. We will show that then there is no irreducible class in $i_{k l}^{-1}[0]$. In fact a class $[x] \in \mathscr{R}\left(f^{l}\right)$ is irreducible iff $p_{i}$ does not divide $x_{i}$ for all $i=1, \ldots, r$. Now for an irreducible class

$$
i_{k l}[x]=\left[p^{\sigma_{1}} / p^{\tau_{1}} \cdot x_{1}, \ldots, p^{\sigma_{r}} / p^{\tau_{r}} \cdot x_{r}\right]=[0] \in \mathrm{Z}_{p_{1}^{a_{1}}} \oplus \cdots \oplus \mathrm{Z}_{p_{r}^{a_{r}}}=\mathscr{R}\left(f^{l}\right)
$$

$\Longleftrightarrow p^{a_{i}} \mid p^{\sigma_{i}-\tau_{i}} \Longleftrightarrow a_{i} \leq \sigma_{i}-\tau_{i}$. But this is impossible for $i=1, \ldots, s$.

Thus the sum reduces to

$$
\sum=\sum_{0}=p_{1}^{\sigma_{1}} \ldots p_{s}^{\sigma_{s}} p_{s+1}^{a_{s+1}} \ldots p_{r}^{a_{r}}=\operatorname{gcd}(k, m) .
$$

In contrast to the nice formula in the previous theorem, if in a decomposition

$$
G=\pi_{1} \bar{X}=\left(\mathrm{Z}_{p_{1}^{a_{11}}} \oplus \cdots \oplus \mathrm{Z}_{p_{1}}^{a_{1 u_{1}}}\right) \oplus \cdots \oplus\left(\mathrm{Z}_{p_{r}^{a_{r 1}}} \oplus \cdots \oplus \mathrm{Z}_{p_{r}}^{a_{r u_{r}}}\right)
$$

we have $u_{j} \geq 2$ then the formula for $N F_{n}^{G}(f)$ becomes more complex.

TheOREM 5.8. Let $G=\pi_{1} X=\mathrm{Z}_{p^{a}} \oplus \cdots \oplus \mathrm{Z}_{p^{a}}$ (u summands) where $p$ denotes a prime. Then for $k=p^{\sigma}$ where $\sigma \geq a$,

$$
N F_{p^{\sigma}}^{G}(f)=\left(p^{a u}+\left(p^{a u}-p^{(a-1) u}\right) \cdot \frac{p^{\sigma-a+1}-p}{p-1}\right) \cdot p^{a u} .
$$

Proof. We consider $[x] \in \mathscr{R}\left(f^{p^{\tau}}\right)$ for $\tau=0, \ldots, \sigma$. We write $x_{j}=p^{c_{j}} x_{j}^{\prime}$ where $p$ does not divide $x_{j}^{\prime}$. Then by Corollary 5.5

- $[x]$ is irreducible $\Longleftrightarrow l=1$ or $c_{j}=0$ for a $j=1, \ldots, u$

$-i_{p^{\sigma}, p^{\tau}}[x]=0 \Longleftrightarrow c_{j} \geq \sigma-a+\tau$ for all $j=1, \ldots, u$.

Let us notice that two above conditions imply $0 \geq \sigma-a+\tau$ hence $\sigma-a \geq \tau$. Thus the contribution to the sum can be nonzero only for $0 \leq \tau \leq \sigma-a$. Moreover the last inequality implies $i_{p^{\sigma}, p^{\tau}}[x]=0$ for all $[x] \in \mathscr{R}\left(f^{p^{\tau}}\right)$. Now

$$
\left\{[x] \in \mathscr{I} \mathscr{R}\left(f^{p^{\tau}}\right) ; i_{p^{\sigma}, p^{\tau}}[x]=0\right\}=\mathscr{I} \mathscr{R}\left(f^{p^{\tau}}\right)
$$


hence

$$
N F_{p^{\sigma}}^{G}(f)=\sum_{\tau=0}^{\sigma-a} \# \mathscr{I} \mathscr{R}\left(f^{p^{\tau}}\right) \cdot p^{\tau} .
$$

For $\tau=0$ all the classes are irreducible, hence $\# \mathscr{I} \mathscr{R}\left(f^{1}\right)=\left(p^{a}\right)^{u}=p^{a u}$.

Let $\tau=1, \ldots, \sigma$. If $[x] \in \mathscr{R}\left(f^{p^{\tau}}\right)$ is irreducible then $c_{j}=0$ for some $j=1, \ldots, u$. There are exactly $p^{a u}-p^{(a-1) u}$ such elements, which implies $\# \mathscr{I} \mathscr{R}\left(f^{p^{\tau}}\right)=p^{a u}-p^{(a-1) u}$. Finally we get

$$
\begin{aligned}
N F_{p^{\sigma}}^{G}(f) & =\left(\sum_{\tau=0}^{\sigma-a} \# \mathscr{I} \mathscr{R}\left(f^{p^{\tau}}\right) \cdot p^{\tau}\right) \cdot p^{a u} \\
& =\left(p^{a u}+\sum_{\tau=1}^{\sigma-a}\left(p^{a u}-p^{(a-1) u}\right) p^{\tau}\right) \cdot p^{a u} \\
& =\left(p^{a u}+\left(p^{a u}-p^{(a-1) u}\right) \cdot \frac{p^{\sigma-a+1}-p}{p-1}\right) \cdot p^{a u} .
\end{aligned}
$$

\section{Periodic points of equivariant maps}

In the previous section we assumed, for the simplicity of the theory, that all the Reidemeister classes were essential. Now we will demonstrate, on the example $G=\mathrm{Z}_{p}$, that some formulae are available without this assumption.

Let $X$ be a simply-connected polyhedron with a free action of the group $Z_{p}$ ( $p$ is a prime). Let $f: X \rightarrow X$ be an equivariant map. We assume that the action of $\pi_{1} \bar{X}$ on $H_{*}(X, \mathrm{Q})$ is trivial. Then, for a fixed natural number $k$, all elements in $\mathscr{O} \mathscr{R}\left(\bar{f}^{k}\right)=\mathscr{R}\left(\bar{f}^{k}\right)=Z_{p}$ are either all essential or all inessential. We will derive formulae for $N F_{p^{\sigma}}^{\mathrm{Z}_{p}}(f)$.

We recall that $\mathscr{R}\left(\bar{f}^{k}\right)=\mathrm{Z}_{p}$ and $i_{p^{\sigma}, p^{\tau}}[x]=\left[p^{\sigma-\tau} \cdot x\right]$. This implies that:

(1) all the classes in $\mathscr{O} \mathscr{R}\left(\bar{f}^{1}\right)=\mathscr{R}\left(\bar{f}^{1}\right)=\mathrm{Z}_{p}$ are irreducible while for $\sigma \geq 1$,

(2) $[0] \in \mathscr{R}\left(\bar{f}^{p^{\sigma}}\right)$ is reducible and the remaining $p-1$ classes in $\mathscr{R}\left(\bar{f}^{p^{\sigma}}\right)$ are irreducible.

Proposition 6.1. Under the above assumptions for every $\sigma \geq 1$ we have

$$
N F_{p^{\sigma}}^{Z_{p}}(f)=p+\sum_{\tau}\left(p^{\tau+2}-p^{\tau+1}\right)
$$

where the summation runs over the set $\left\{\tau \in \mathrm{Z} ; 0 \leq \tau \leq \sigma-1, L\left(f^{p^{\tau}}\right) \neq 0\right\}$ 
Proof. It follows from the above considerations that for $\sigma>\tau$

$$
\mathscr{I} \mathscr{E} O \mathscr{R}\left(f^{p^{\tau}}\right) \cap i_{p^{\sigma}, p^{\tau}}^{-1}[0]= \begin{cases}0 & \text { for } L\left(f p^{p^{\tau}}\right)=0, \\ p & \text { for }(\sigma>\tau=0) \text { and } L\left(f^{1}\right) \neq 0, \\ p-1 & \text { for }(\sigma \geq \tau \geq 1) \text { and } L\left(f^{1}\right) \neq 0 .\end{cases}
$$

First we consider the case $L\left(f^{1}\right) \neq 0$. We will show that then the family

$$
\mathscr{A}=\bigcup_{\tau \mid \sigma \tau<\sigma} \mathscr{I} \mathscr{E} \mathscr{O R}\left(f^{p^{\tau}}\right)
$$

is (the unique) minimal preceding system (see Remark 4.7) for

$$
\mathscr{B}=\bigcup_{\tau \mid \sigma} \mathscr{O} \mathscr{R}\left(f^{p^{\tau}}\right) \cap i_{p^{\sigma}, *}^{-1}[0] .
$$

First we notice that $\mathscr{B}=\bigcup_{\tau \mid \sigma, \tau<\sigma} \mathscr{O} \mathscr{R}\left(f^{p^{\tau}}\right)$, since $i_{p^{\sigma}, p^{\tau}}=0$ for $\sigma<\tau$ and $i_{p^{\sigma}, p^{\sigma}}=$ id. Now each preceding system of $\mathscr{B}$ must contain $\mathscr{A}$.

It remains to show that each essential class $B \in \mathscr{B}$ reduces to $\mathscr{A}$. This is obvious if $B$ is irreducible. On the other hand each reducible class reduces to $\mathscr{R}\left(f^{1}\right) \subset \mathscr{A}$, since $L\left(f^{1}\right) \neq 0$ implies that all classes in $\mathscr{R}\left(f^{1}\right)$ are essential.

Now

$$
\begin{aligned}
N F_{p^{\sigma}}^{Z_{p}}\left(f^{p^{\sigma}}\right) & =\left(\sum_{\tau=0}^{\sigma-1} \# \mathscr{I E} \mathcal{C} \mathscr{R}\left(f^{p^{\tau}}\right) \cdot p^{\tau}\right) \cdot p \\
& =\left(p+\sum_{1 \leq \tau \leq \sigma-1 ; L\left(f^{p^{\tau}}\right) \neq 0}(p-1) \cdot p^{\tau}\right) \cdot p \\
& =\left(1+\sum_{0 \leq \tau \leq \sigma-1 ; L\left(f^{p^{\tau}}\right) \neq 0}\left(p^{\tau+1}-p^{\tau}\right)\right) \cdot p \\
& =p+\sum_{0 \leq \tau \leq \sigma-1 ; L\left(f^{p^{\tau}}\right) \neq 0}\left(p^{\tau+2}-p^{\tau+1}\right) .
\end{aligned}
$$

Now we assume that $L\left(f^{1}\right)=0$. If moreover all $L\left(f^{p^{\tau}}\right)=0$ then all involving classes are inessential and $N F_{G}\left(f^{p^{\sigma}}\right)=0$ as the sum is taken over the empty set. Hence we assume that $L\left(f^{p^{\tau}}\right) \neq 0$ for a $\tau$. Then following the above we get for $\sigma>\tau$

$$
\mathscr{\mathscr { C } O \mathscr { O }} \mathscr{R}\left(f^{p^{\tau}}\right) \cap i_{p^{\sigma}, p^{\tau}}^{-1}[0]= \begin{cases}0 & \text { for } L\left(f^{p^{\tau}}\right)=0, \\ p-1 & \text { for } L\left(f^{p^{\tau}}\right) \neq 0 .\end{cases}
$$




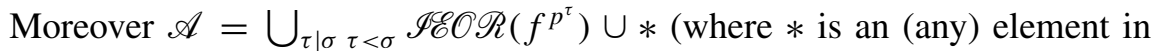
$\left.\mathscr{R}\left(f^{1}\right)\right)$ is a minimal preceding system for $\mathscr{B}=\bigcup_{\tau \mid \sigma} \mathscr{O R}\left(f^{p^{\tau}}\right) \cap i_{p^{\sigma}, *}^{-1}[0]$. This implies

$$
\begin{aligned}
& N F_{G}\left(f^{p^{\sigma}}\right)=\left(\left(\sum_{\tau=0}^{\sigma-1} \# \mathscr{I} \mathscr{C} \mathscr{O} \mathscr{R}\left(f^{p^{\tau}}\right) \cdot p^{\tau}\right)+1\right) \cdot p \\
& =\left(\left(\sum_{0 \leq \tau \leq \sigma-1 ; L\left(f^{p^{\tau}}\right) \neq 0}(p-1) \cdot p^{\tau}\right)+1\right) \cdot p \\
& =p+\sum_{0 \leq \tau \leq \sigma-1 ; L\left(f p^{\tau}\right) \neq 0}\left(p^{\tau+2}-p^{\tau+1}\right) .
\end{aligned}
$$

COROLLARY 6.2. Let $X$ be a simply-connected polyhedron with a free action of the group $\mathrm{Z}_{p}$ ( $p$ is a prime). Let $f: X \rightarrow X$ be an equivariant map. We assume that the action of $\mathrm{Z}_{p}=\pi_{1} \bar{X}$ on $H_{*}(X, \mathrm{Q})$ is trivial.

(1) If there are infinitely many $\sigma \geq 0$ satisfying $L\left(f^{p^{\sigma}}\right) \neq 0$ then

$$
\lim \sup \frac{\# \operatorname{Fix}\left(f^{n}\right)}{n} \geq p-1 .
$$

(2) If $L\left(f^{p^{\sigma}}\right) \neq 0$ for all $\sigma \geq 0$ then

$$
\lim \sup \frac{\# \operatorname{Fix}\left(f^{n}\right)}{n} \geq p .
$$

Proof. Let $L\left(f^{p^{\sigma}}\right) \neq 0$. Since all the summands (brackets) in Theorem 6.1 are nonnegative we get the inequality:

$$
N F_{p^{\sigma+1}}^{Z_{p}}(f)=p+\sum_{\tau}\left(p^{\tau+2}-p^{\tau+1}\right) \geq p^{\sigma+2}-p^{\sigma+1} .
$$

Now if the set $\left\{\sigma \in \mathbf{N} ; L\left(f^{p^{\sigma}}\right) \neq 0\right\}$ is infinite then

$$
\begin{aligned}
\limsup _{n} \frac{\# \operatorname{Fix}\left(f^{n}\right)}{n} & \geq \limsup _{n} \frac{N F_{n}^{z_{p}}(f)}{n} \geq \underset{\sigma}{\limsup } \frac{N F_{p^{\sigma+1}}^{z_{p}}(f)}{p^{\sigma+1}} \\
& \geq \limsup _{\sigma} \frac{p^{\sigma+2}-p^{\sigma+1}}{p^{\sigma+1}}=p-1
\end{aligned}
$$

where $\sigma$ runs through the (infinite) set $\left\{\sigma \in \mathrm{N} ; L\left(f^{p^{\sigma}}\right) \neq 0\right\}$. 
Now we assume that $L\left(f^{p^{\sigma}}\right) \neq 0$ for all $\sigma \geq 0$. Then

$$
N F_{p^{\sigma}}^{Z_{p}}(f)=p+\sum_{0 \leq \tau \leq \sigma-1}\left(p^{\tau+2}-p^{\tau+1}\right)=p^{\sigma+1}
$$

hence

$$
\limsup _{n} \frac{\# \operatorname{Fix}\left(f^{n}\right)}{n} \geq \underset{\sigma}{\limsup } \frac{N F_{p^{\sigma}}^{z_{p}}(f)}{p^{\sigma}}=\underset{\sigma}{\limsup } \frac{p^{\sigma+1}}{p^{\sigma}}=p .
$$

In the next theorem we provide a condition which needs to be verified for only one iteration of a given map.

THEOREM 6.3. Let $X$ be a simply-connected finite polyhedron with a free action of a finite group $G$ and $f: X \rightarrow X$ be a $G$-equivariant map. Assume that the action of $G$ on $H_{*}(X ; \mathrm{Q})$ is trivial.

If there exist a prime $p$ dividing the order $\# G$ and there is an $a \in \mathrm{N}$ such that

$$
L\left(f^{p^{a}}\right) \varsubsetneqq 0 \bmod \left(p^{a+1}\right)
$$

then $f$ has infinitely many periodic points and

$$
\lim \sup \frac{\# \operatorname{Fix}\left(f^{n}\right)}{n} \geq p .
$$

Proof. First of all observe that we can restrict the action to a cyclic subgroup $\mathrm{Z}_{p} \subset G$. It is also free action, $\mathrm{Z}_{p}$ acts trivially on the homology groups and $f$ is $Z_{p}$-equivariant.

We show that the hypothesis (2) of Corollary 6.2 is satisfied. By the Dold congruences (see [15][Chapter III] for a purely algebraic proof), we have $L\left(f^{p^{a}}\right) \cong L\left(f^{p^{a+1}}\right) \bmod \left(p^{a+1}\right)$ for any $a \geq 0$. From that it follows that $L\left(f^{p^{a+1}}\right)=L\left(f^{p^{a}}\right)+k_{1} p^{a+1} ¥ 0 \bmod \left(p^{a+1}\right)$. Next $L\left(f^{p^{a+2}}\right)=L\left(f^{p^{a+1}}\right)+$ $k_{2} p^{a+2}=L\left(f^{p^{a}}\right)+k_{1} p^{a+1}+k_{2} p^{a+2} ¥ 0 \bmod \left(p^{a+1}\right)$. Continuing this argument we get $L\left(f^{p^{a+i}}\right) \not 0 \bmod \left(p^{a+i}\right)$ for every $i \geq 0$, which shows that the condition (2) of Corollary 6.2 is satisfied for $\sigma \geq a$.

REMARK 6.4. Note that for a free action of a finite group $G$ on a polyhedron $X$ and a $G$-equivariant self-map $f: X \rightarrow X$ we have $L(f) \cong 0 \bmod (\# G)$ which can be proved by the use of the homomorphism induced on chains and the Hopf lemma. It follows that in our assumption $L\left(f^{p^{a}}\right) \neq 0 \quad \bmod \left(p^{a+1}\right)$ the case $a=0$ is excluded.

Remark 6.5. Observe also that in the assumptions of Corollary 6.2 and Theorem 6.3 we do not require that the sequence $L\left(f^{n}\right)$ is unbounded as was 
assumed in [2] and [19]. On the other hand the assumption of Corollary 6.2 and Theorem 6.3 is obviously satisfied if $\left|L\left(f^{n}\right)\right| \stackrel{n \rightarrow \infty}{\longrightarrow} \infty$.

\section{Applications}

In this section we present a few cases in which our results can be applied. We would like to emphasize that they are only particular examples which could be discussed in a more general form but it would be beyond the main scope of the work.

The case when $X=S^{n}$ is the sphere with a free action of a finite group $G$ was discussed and completely described in [14]. It is worth emphasizing that then (see [14] for more details):

a) For any finite group $G$, free action and an equivariant map $f: S^{n} \rightarrow$ $S^{n}$ the quotient map $\bar{f}: S^{n} / G \rightarrow S^{n} / G$ is essentially reducible if $|\operatorname{deg}(f)|>1$.

b) For any finite group free action and an equivariant map $f: S^{n} \rightarrow$ $S^{n}$, all its iterations $f^{k}$ and compositions $f^{k} g, g \in G$ are essential if $|\operatorname{deg}(f)|>1$ (we do not need the assumption that the induced action on homology spaces is trivial).

c) If a finite abelian group $G$ acts free on $S^{n}$ then it is cyclic as follows from the Milnor theorem.

Consequently, for a free action of $G=\mathrm{Z}_{m}$ on $S^{n}$ and an equivariant map $f: S^{n} \rightarrow S^{n}$ with $|\operatorname{deg}(f)|>1$ we have $N F_{k}^{G}(f)=k^{\prime} \cdot m$, thus \# $\operatorname{Fix}\left(f^{k}\right) \geq$ $k^{\prime} \cdot m$.

Let us next consider the product of spheres

$$
X=S^{d_{1}} \times S^{d_{2}} \times \cdots \times S^{d_{r}}
$$

By the Künneth formula the cohomology ring $H^{*}(X ; \mathrm{Q})$ is an algebra over $\mathrm{Q}$ with generators $\gamma_{i} \in H^{d_{i}}(X ; \mathrm{Q}), \gamma_{i}^{2}=0$, each of which corresponds to the factor $S^{d_{i}}$. Moreover if we assume that all the $d_{i}$ are odd then $X$ is the cohomology rational exterior power, i.e., for the elements $x_{i}:=p_{i}^{*}\left(y_{i}\right)$ the algebra $H^{*}(X ; \mathrm{Q})$ is isomorphic to the exterior algebra $\bigwedge_{\mathrm{Q}}\left(x_{1}, \ldots, x_{r}\right)$, where $p_{i}: X \rightarrow S^{d_{i}}$ is the projection and $y_{i} \in H^{d_{i}}\left(S^{d_{i}} ; \mathrm{Q}\right) \equiv \mathrm{Q}$ is a generator

The fixed points of self-maps of cohomology exterior powers have been studied by Duan [4], then by Graff [9] (cf. also [15], Chapt. 3.4 for details). The main result is the following property [4]: there exists an integral matrix $A_{f}=\bigoplus_{l=1}^{d} A_{f, l}, d=\operatorname{dim} X$, such that for every $n \in \mathrm{N}$

$$
L\left(f^{n}\right)=\operatorname{det}\left(\mathrm{I}-A_{f, n}\right)
$$


The matrix $A_{f}$ is equal to the linear map induced by $H^{*}(f)$ on the quotient space $A^{*}(X):=H^{*}(X ; \mathrm{Q}) / D^{*}(X)$, where $D^{*}(X)$ is the subspace of decomposable elements of the algebra $H^{*}(X ; \mathrm{Q})$. By the definition it is a rational matrix but is not difficult to show that $A_{f}$ is an integral matrix (cf. [4]).

In the case of $X=S^{d_{1}} \times S^{d_{2}} \times \cdots \times S^{d_{r}}$ the coefficients $a_{i, j}$ of the matrix $A_{f, l}$, are given by the formula expressing the image of each generator $\gamma_{i}$ corresponding to $S^{d_{i}}, d_{i}=l$ :

$$
H^{l}(f)\left(\gamma_{i}\right)=\sum_{d_{j}=l} a_{i, j} \gamma_{j}
$$

Note also that in this case the dimension of $A^{*}(X)$, called the rank of the exterior rational power, is equal to $r$.

Let $\lambda_{1}, \lambda_{2}, \ldots, \lambda_{r}$ be all eigenvalues of $A_{f}$.

We are in a position to formulate a theorem which extends the main result of [14].

THEOREM 7.1. Let a finite group $G$ acts freely on the space $X=S^{d_{1}} \times$ $S^{d_{2}} \times \cdots \times S^{d_{r}}, d_{i} \geq 3$ are odd. Let $f: X \rightarrow X$ be an equivariant map and $A_{f}$ the integral matrix associated with $H^{*}(f)$.

Suppose that there exists a prime $p|| G \mid$ such that the spectrum of $A_{f}$ contains no root of unity of order $p^{\tau}, \tau \geq 1$.

Then $f$ has infinitely many periodic points. Moreover we have the estimate

$$
\lim \sup \frac{\# \operatorname{Fix}\left(f^{n}\right)}{n} \geq p .
$$

Proof. If we assumed moreover that the action of $\pi_{1} \bar{X}$ on $H^{*} X$ are trivial then the theorem would follow from Corollary 6.2, (ii). However this assumption was used only to guarantee that all Reidemeister classes of $\bar{f}^{p^{\sigma}}$, for all $n \in \mathrm{N}$, are trivial. Since the last statement is equivalent to $L\left(g f^{p^{\sigma}}\right) \neq 0$ (for all $n \in \mathrm{N}, g \in G$ ), it remains to prove this directly.

First note that for the computation of the Lefschetz number we can use cohomology with complex coefficients $H^{*}(X ; \mathrm{C})=H^{*}(X ; \mathrm{Q}) \otimes \mathrm{C}$. Next, since $f^{n} g=g f^{n}, H^{*}(g) H^{*}\left(f^{n}\right)=H^{*}\left(f^{n}\right) H^{*}(g)$, and consequently $H^{*}(g)$ preserves the generalized eigenspaces of $H^{*}\left(f^{n}\right)$ and so does $H^{*}\left(f^{n}\right)$ with the generalized eigenspaces of $H^{*}(g)$. By the same argument, we have the same property for the linear maps of $A^{*}(X) \otimes \mathrm{C}$. This shows that the matrix $A_{g f^{n}}=A_{f^{n}} A_{g}=A_{f}^{n} A(g)$ has eigenvalues of the form $\lambda_{i}^{n} \mu$, where $\lambda_{i}, 1 \leq$ $i \leq r$ is an eigenvalue of $A_{f}$, and $\mu$ an eigenvalue of $A_{g}$. But all eigenvalues of $H^{*}(g)$, thus of $A_{g}$, are roots of unity of order $p$. For a given $\mu_{j}=\exp \left(\frac{2 \pi i j}{p}\right)$, 
$0 \leq j \leq p-1$, let $\lambda_{i_{1}}, \ldots \lambda_{i_{r_{j}}}$ be all eigenvalues of $A_{f}$ corresponding to the $\mu_{j}$.

From the formula (5) we have

$$
L\left(g f^{n}\right)=\operatorname{det}\left(\mathrm{I}-A_{f}^{n} A(g)\right)=\prod_{j=0}^{p-1} \prod_{1}^{r_{j}}\left(1-\lambda_{i_{j}}^{n} \mu_{j}\right) .
$$

It is enough to show that for every $\sigma$ (or for $\sigma$ sufficiently large) and $n=p^{\sigma}$ we have $L\left(g f^{n}\right) \neq 0$. Now the above product is equal to 0 if and only if at least one of its factors $\left(1-\lambda_{i}^{p^{\sigma}} \mu_{j}\right)$ is zero.

We will show that the last is impossible. In fact $\lambda_{i}^{p^{\sigma}} \mu_{j}=1$ and $\mu_{j}^{p}=1$ imply

$$
1=\left(\lambda_{i}^{p^{\sigma}} \mu_{j}\right)^{p}=\lambda_{i}^{p^{\sigma+1}} \mu_{j}^{p}=\lambda_{i}^{p^{\sigma+1}}
$$

contradicting the assumption that $\lambda_{i}$ is not a root of unity of order being a power of $p$.

This proves the statement of Theorem 7.1.

Remark 7.2. In the above theorem we did not need to assume that the action of $G$ on $H^{*}(X ; \mathrm{Q})$ is trivial.

Remark 7.3. Note that in Theorem 7.1 we do not assume that the sequence $L\left(f^{n}\right)$ is unbounded! If $r=1, X=S^{d}, d$-odd, then $A_{f}=\operatorname{deg}(f) \in \mathrm{Z}$ and our assumption reads that $\operatorname{deg}(f) \neq 1$ if $p$ is odd, or $\operatorname{deg}(f) \neq-1$ if $p=2$. The case $\operatorname{deg}(f)=0$ is excluded, since $\operatorname{deg} f \cong 1 \bmod (p)$ for an equivariant map of a sphere due to a $Z_{p}$ version of the Borsuk-Ulam theorem (see [14] for more details). Consequently for $r=1$ the assumption of Theorem 7.1 is equivalent to $\operatorname{deg}(f) \notin\{-1,0,1\}$, but the latter is exactly the assumption of the main theorem of [14].

ReMARK 7.4. For some recent results and the literature on actions of finite groups on the space $X=\left(S^{d}\right)^{r}$ we refer the reader to [1].

Remark 7.5. Note that the statement of Theorem 7.1 holds for an equivariant map $f$ of any simply-connected space $X$ which is rational exterior power and which admits a free action of a finite group $G$.

\section{REFERENCES}

1. Adem, A., Davis, J., and Ünlü, Ö., Fixity and free group actions on products of spheres, Comment. Math. Helv. 79 (2004), 758-778.

2. Babenko, I. K., Bogaty̆ , S. A., Behavior of the index of periodic points under iterations of a mapping (Russian), Izv. Akad. Nauk SSSR (Mat.) 55 (1991), 3-31; transl.: Math. USSR Izv. 38 (1992), 1-26. 
3. Bredon, G., Introduction to Compact Transformation Groups, Pure Appl. Math. 46, Academic Press, New York 1972.

4. Duan, H., The Lefschetz number of iterated maps, Topology Appl. 67 (1995), 71-79.

5. Fadell, E., and Wong, P., On deforming G-maps to be fixed point free, Pacific J. Math. 132 (1988), 277-281.

6. Fel'shtyn, A., Hill, R., and Wong, P., Reidemeister numbers of equivariant maps, Topology Appl. 67 (1995), 119-131.

7. Ferrario, D. L., A fixed point index for equivariant maps, Topol. Methods Nonlinear Anal. 13 (1999), 313-340.

8. Ferrario, D. L., A note on equivariant fixed point theory, pp. 287-300 in: Handbook of Topological Fixed Point Theory, Springer, Dordrecht 2005.

9. Graff, G., Minimal periods of maps of rational exterior spaces, Fund. Math. 163 (2000), 99-115.

10. Graff, G., and Jezierski, J., On the growth of the number of periodic points for smooth selfmaps of compact manifolds, Proc. Amer. Math. Soc. 135 (2007), 3249-3254.

11. Jezierski, J., Wecken theorem for periodic points, Topology 42 (2003), 1101-1124.

12. Jezierski, J., Wecken's theorem for periodic points in dimension at least 3, Topology Appl. 153 (2006), 1825-1837.

13. Jezierski, J., and Marzantowicz, W., Homotopy minimal periods for nilmanifold maps, Math. Z. 239 (2002), 381-414.

14. Jezierski, J., and Marzantowicz, W., A symmetry of sphere map implies its chaos, Bull. Braz. Math. Soc. (N.S.) 36 (2005), 205-224.

15. Jezierski, J., and Marzantowicz, W., Homotopy Methods in Topological Fixed and Periodic Points Theory, Topological Fixed Point Theory and Appl. 3, Springer, Dordrecht 2006.

16. Jiang, B., Lectures on Nielsen Fixed Point Theory, Contemp. Math. 14, Amer. Math. Soc., Providence, RI 1983.

17. Jiang, B., Estimation of the number of periodic orbits, Pacific J. Math. 172 (1996), 151-185.

18. Jiang, B., and Llibre, J., Minimal sets of periods for torus maps, Discrete Contin. Dynam. Systems 4 (1998), 301-320.

19. Shub, M., and Sullivan, D., A remark on the Lefschetz fixed point formula for differentiable maps, Topology 13 (1974), 189-191.

20. Weber, J., Equivariant Nielsen invariants for discrete groups, Pacific J. Math. 231 (2007), 239-256.

21. Wong, P., Equivariant Nielsen numbers, Pacific J. Math. 159 (1993), 153-175.

FACULTY OF INFORMATICS AND MATHEMATICS WARSAW UNIVERSITY OF LIFE SCIENCES

UL. NOWOURSYNOWSKA 159

02-787 WARSZAWA

POLAND

E-mail: jerzy_jezierski@sggw.pl
FACULTY OF MATHEMATICS AND COMPUTER SCI. ADAM MICKIEWICZ UNIVERSITY OF POZNAŃ UL. UMULTOWSKA 87

61-614 POZNAŃ

POLAND

E-mail: marzan@amu.edu.pl 